\title{
Hemorrhagic complications in dermatologic surgery
}

\author{
Christopher G. Bunick and Sumaira Z. Aasi \\ Department of Dermatology, Yale University, New Haven, Connecticut
}

\begin{abstract}
The ability to recognize, manage, and, most importantly, prevent hemorrhagic complications is critical to performing dermatologic procedures that have safe and high quality outcomes. This article reviews the preoperative, intraoperative, and postoperative factors and patient dynamics that are central to preventing such an adverse outcome. Specifically, the role that anticoagulants and antiplatelet agents, hypertension, and other medical conditions play in the development of postoperative hemorrhage are discussed. In addition, this article provides practical guidelines on managing bleeding during and after surgery.
\end{abstract}

\section{Keywords}

anticoagulants; hematoma; hemorrhage

\section{Introduction}

Hemorrhage is often the perilous initiator in the series of complications well known to surgeons as the terrible tetrad. Postoperative bleeding can lead to the development of a hematoma, which subsequently can lead to other grave surgical consequences, such as wound infection, dehiscence, and necrosis. Such adverse events not only cause anxiety and mental stress for the patient and the physician, but also may ultimately result in a poor outcome with suboptimal scar formation. There are multiple factors that potentially affect whether a patient experiences bleeding during or after a surgical procedure: age of the patient, medications, medical comorbidities, including bleeding diatheses, and postoperative activity levels, to name a few. The overall safety of dermatologic surgery, whether comparing basic excisional procedures versus Mohs micrographic surgery (MMS) or an outpatient office versus hospital-based setting, is supported by multiple prospective and retrospective analyses (1-4). Even though these studies demonstrate the overwhelming safety of the procedures performed by dermatologic surgeons, albeit using slightly different study parameters (e.g., variability in the definitions of mild, moderate, or severe complications), they suggest that when complications do arise, there is a strong probability it will be related to some magnitude of bleeding. In a prospective study, assessing six core complications (post-operative hemorrhage, hematoma formation, wound dehiscence, wound infection, flap necrosis, and skin graft necrosis), Cook and Perone identified only 22 
complications out of 1343 MMS cases (1.64\% overall complication rate) (2). These 22 complications stratified into seven hematoma events and two postoperative hemorrhage events; in other words, $40 \%$ of the complications were related to a bleeding event (2). Similarly, Kimyai-Asadi and colleagues found that out of 3937 MMS cases in Houston, Texas (2397 outpatient, 1540 hospital-based), only one could be classified as "severe" (defined in this study as life-threatening or potential compromise of organ function): a case of post-operative gastrointestinal hemorrhage due to naproxen medication (3). Thus, the data suggests that anyone performing dermatologic procedures must have the skill set to deal with bleeding events despite the overall low incidence of such complications.

The goal of this article is to present practical ways in which a dermatologic surgeon can recognize, manage, and prevent hemorrhagic complications of cutaneous surgery. The discussion will proceed chronologically from necessary preoperative considerations, to intraoperative management of bleeding, and finally to hemorrhagic issues in the postoperative patient.

\section{Pre-operative considerations to cutaneous surgery}

Prior to placing a scalpel to skin, a complete medical history must be obtained from the patient, including a review of medical problems, medications (prescribed, over-the-counter, and herbals), surgical history, family history, and allergies. An appropriate and thorough preoperative evaluation lays the foundation for procedures that have safe and high-quality outcomes. With regards to hemorrhage, there are four major categories to address with a patient in order to evaluate pre-operative bleeding risk: (i) anticoagulation; (ii) hypertension; (iii) other medical comorbidities; and possibly, (iv) anatomy.

\section{Anticoagulation}

Because of the importance of maintaining hemostasis in surgery, anticoagulants are often the most essential medications a surgeon identifies in the preoperative evaluation. The debate on whether to have patients stop taking aspirin, NSAIDs, warfarin, or other newer antiplatelet agents prior to cutaneous surgery has somewhat subsided. The overwhelming evidence, and the risk-to-benefit ratio point in general toward the continuation of these agents pre-, intraand postoperatively. Between 1996 and 2004, six controlled studies (two retrospective, four prospective) investigated the risk of bleeding complications in patients taking or not taking anticoagulants during cutaneous surgery (4-9). In their retrospective review, specifically looking at the impact of anticoagulants on 12,000 procedures, Otley and colleagues found a severe complication rate of $1.6 \%$ for patients on some type of anticoagulant versus $0.7 \%$ for control patients, again confirming the overall relatively low rate of complications in dermatologic procedures. They report on 11 patients categorized as having "severe complications" (defined as potential significant threat to wound or patient; significant intraor post-operative hemorrhage; wound bleeding greater than 1-hour duration not alleviated with pressure; acute hematoma; necrosis of flap or graft; wound dehiscence greater than 2 $\mathrm{mm})$. Of these 11 patients, nine $(81 \%)$ had a bleeding complication ( 8 postoperative bleeding and 1 hematoma); the other two patients experienced graft necrosis. Billingsley et al. looked at patients undergoing MMS and found no significant difference in postoperative bleeding complications in patients taking anticoagulants, though there was a non-significant 
trend toward increased bleeding in patients taking warfarin (7). All six of these studies, except that by Kargi and colleagues, did not find increased bleeding complications among patients undergoing cutaneous surgical procedures while taking anticoagulants. These six studies were then analyzed by a meta-analysis performed by Lewis and Dufresne (10). This meta-analysis involved 122 patients taking warfarin, 472 patients on aspirin, and 779 controls undergoing dermatologic surgery for benign and malignant lesions. The study found a sixfold increased risk of moderate to severe bleeding complications in patients taking warfarin compared with controls ( $12.3 \%$ vs. $2.1 \%$, respectively). Patients taking aspirin had a non-significantly increased risk of bleeding with a $4 \%$ rate of moderate to severe complications. Limitations in comparing these studies both individually and by metaanalysis have been the slightly different and fairly qualitative definitions for mild, moderate, or severe complications. Finally, clopidogrel is a newer anticoagulant with extensive use particularly in patients who have undergone intracoronary stent placement for myocardial infarction, and has synergistic antiplatelet effects when combined with aspirin. The effect of clopidogrel on bleeding complications in dermatologic surgery has not been well-studied, but our own experience and published data from the general medical literature suggest that the combination of aspirin and clopidogrel is similar to warfarin in terms of bleeding risk (11).

Perhaps the measure of "severe," "serious," or "life-threatening" complications with respect to anticoagulants in dermatologic surgery is better understood through studies investigating thrombotic complications related to withholding these medications in patients undergoing surgical procedures. Although stopping these medications was an accepted practice among dermatologic and plastic surgeons 10-20 years ago, several pharmacologic and physiologic aspects of these medications must be recognized when considering a temporary cessation of anticoagulation. First, aspirin and clopidogrel cause an irreversible inhibition of platelet function that can only be recovered by the synthesis of new platelets; the lifespan of circulating platelets is 7-10 days. Warfarin does not affect platelets, so it actually does not have as much of an effect on intra- or immediate postoperative bleeding. Instead, warfarin interferes with the formation of a fibrin plug, which in turns affects the clotting cascade and often impairs hemostasis 72 to 96 hours post surgery. Specifically, it inhibits the synthesis of serum coagulation proteins, such as factor II, VII, IX, and X; the half-life of these factors in serum ranges from 1 to 3 days. Thus, recovery of platelet and clotting factor function requires at least 4-7 days of therapy cessation prior to any scheduled procedure. Second, resumption of the therapeutic antithrombotic effect requires another 4-7 days after the anticoagulant is initiated due to the gradual decay of serum clotting factors or inhibition of platelet function. Third, compensatory factors induced by chronic anticoagulant treatment, such as decreased levels of protein $\mathrm{C}$ and $\mathrm{S}$ or increased activity of other clotting factors, may lead to a transient hypercoagulable state when these medications are interrupted $(12,13)$. It is therefore likely that interruption of anticoagulant therapy for a 1-2-hour surgical procedure could result in up to 2 weeks of increased risk of systemic thrombosis.

Several case reports in the literature have described catastrophic thrombotic events when therapeutic anticoagulation was held for cutaneous surgery. Schanbacher and Bennett were the first to report in the dermatologic literature on two patients who suffered acute thrombotic stroke when warfarin was held for MMS of facial basal cell carcinoma (14). In 
both cases, warfarin was held for one week prior to surgery, restarted the day following the procedure, and the thrombotic complications occurred 3-4 days after the procedure. Of note, anticoagulation in both patients was interrupted under consultation with the patient's internist or cardiologist. Holding therapy with antiplatelet agents can also have deleterious effects, as reported in one case of major pulmonary embolus and one case of prosthetic aortic valve thrombosis when dual anticoagulant therapy (aspirin and ticlopidine in one case and clopidogrel and ardeparin, a low-molecular weight heparin, in the other, respectively) was stopped 7 days prior to surgery (15). In both of these reports, the thrombotic event occurred 2-3 days after surgery. To estimate the incidence of these rare adverse events, Kovich and Otley reported findings from a survey of 168 responding physicians belonging to the American College of Mohs Micrographic Surgery and Cutaneous Oncology (16). These physicians reported 49 thrombotic complications in 46 patients ( 3 deaths, 24 strokes, 3 cerebral emboli, 5 myocardial infarctions, 8 transient ischemic attacks, 3 deep venous thromboses, 2 pulmonary emboli, and 1 retinal artery occlusion). Fifty-four percent of the thrombotic complications were attributed to withholding of warfarin and $39 \%$ attributed to withholding of aspirin. The authors calculated an estimated thrombotic risk of one event per $\sim 12,800$ operations and suggested that at least one thrombotic event would occur per career in $50 \%$ of dermatologic surgeons. Kirkorian and colleagues (17) performed in 2005 a survey assessing anticoagulant and antiplatelet management habits in cutaneous surgery practices, and compared their findings with those of a similar survey performed three years prior (18). The largest difference they identified was that only 44\% (down from 80\%) of Mohs surgeons still discontinued warfarin therapy some of the time - this marked a notable shift in the paradigm of anticoagulant use during cutaneous surgery. Interestingly, in their study, they also report that 82 Mohs surgeons had personally seen 126 thrombotic events (15 deaths, 39 strokes, 19 myocardial infarctions, 25 transient ischemic attacks, 7 deep venous thromboses, 4 pulmonary emboli, and 17 unstable angina). A more systematic review of published cases from the dental literature found 542 cases in which warfarin therapy was suspended, and five cases of thrombotic complications, 4 of which were fatal (19). Given that the literature in general demonstrates that bleeding complications have only minimal clinical significance, whereas thrombotic complications, albeit rare, may result in devastating consequences, such as death or permanent neurologic disability, dermatologic surgeons often choose to have patients remain on anticoagulants in the vast majority of dermatologic surgery procedures. While the risk-to-benefit ratio of clopidogrel has not been defined in these studies, the American College of Cardiology recommends that patients taking clopidogrel after myocardial infarction should remain on their anticoagulant therapy during minor surgical procedures, such as local skin surgery (20). A recent prospective study by Kramer and colleagues found no significant difference in complication rate for 32 patients undergoing cutaneous operation while taking clopidogrel or clopidogrel plus aspirin compared with 2329 patients taking aspirin alone, warfarin alone, or no anticoagulant or antiplatelet agent (21). Situations where anticoagulant medications can be interrupted in dermatologic surgery include cases where a patient is taking aspirin for primary prevention (with no prior history of a thrombotic event) or when a patient is taking aspirin or other NSAIDs for pain relief, rather than for the anticoagulant effect. In this latter case, we advise the patient to suspend NSAIDs for 1 week before and 2 days after the surgical procedure. Pain relief in the perioperative period can then be managed with acetaminophen. Finally, in 
patients on warfarin in whom a significantly large or complex surgical procedure is anticipated, it would be judicious to check the preoperative international normalized ratio (INR) to rule out supratherapeutic INR (>3.5) and discuss management considerations with the patient's cardiologist.

In addition to therapeutic anticoagulants, several herbal or over-the-counter (OTC) medications can have intended or unintended effects on the coagulation pathway. It is important for the surgeon to be cognizant of these medications. Because patients may not be aware of the anticoagulant effects, the surgeon must specifically question patients about the use of these traditional and nontraditional medications. Several common OTC medications contain aspirin or ibuprofen as the active ingredients, such as Alka-Seltzer@ (Bayer, Morristown, NJ, USA) and Advil® Cold and Sinus (Pfizer, New York, NY, USA), respectively. Herbal medications and supplements have also been implicated in several cases of spontaneous or postoperative bleeding, including one report of serious hemorrhage requiring transfusion after uncomplicated laparoscopic cholecystectomy in an otherwise healthy 36-year-old man taking Ginkgo biloba supplements (22). These reports of hemorrhagic complications are supported by mechanistic studies showing that ginkgo extracts reduce blood viscosity and inhibit platelet activating factor. Isolated case reports and in vitro studies of platelet activation and coagulation factors have also implicated garlic, ginseng, ginger, feverfew, vitamin E, and saw palmetto in exacerbated operative and postoperative bleeding $(23,24)$. The prevalence of dietary supplement use in the dermatologic surgery population should not be underestimated; Collins and Dufresne found in a 2002 study that $49 \%$ of MMS patients were taking dietary supplements, and $65 \%$ of them failed to even report it on a pre-operative questionnaire (25). This latter fact begs the following question: how much of the dermatologic literature evaluating bleeding complications in the setting of anticoagulant or antiplatelet use accounts properly for dietary supplement use? Even if a large percentage of the patients in all the studies cited above were unknowingly on herbal or dietary supplements, the lack of life-threatening complications and low overall complication rates argue that herbal supplement impact on the vast majority of cutaneous surgical procedures and its associated hemorrhagic risks is minimal. In their analysis of multiple antithrombotic agents, Shimizu and colleagues included vitamin E, fish oil, and gingko: of the 32 patients on one or more of these dietary supplements (some in combination with aspirin, clopidogrel, or warfarin), none experienced a bleeding complication (26). However, for all these agents, definitive studies of bleeding risk have not been performed, and it remains difficult to assess whether the published case reports represent causality or coincidence. Nevertheless, because these supplements are used primarily for prevention rather than treatment and because there is currently no known risk of stopping these products, it is recommended that patients discontinue the use of all herbal products 1 week prior to surgery.

\section{Hypertension}

The measurement of a patient's blood pressure is a simple and vital part of any visit to a physician's office, including visits to the Mohs surgery suite. For many years, elevated blood pressure has been identified as a risk factor for hemorrhagic complications in cutaneous surgery, particularly postoperative wound bleeding and hematoma formation. An 
early example of this principle came from a study by Straith and colleagues in the 1970s, where they found patients with blood pressures $150 / 100 \mathrm{mmHg}$ or higher prior to rhytidectomy were 2.6 times more likely to experience postoperative wound hematomas than patients with normal blood pressure (27). Problems that may arise due to postoperative wound bleeding or hematoma are wound dehiscence, increased infection risk, and necrosis of tissue (28). With regards to the latter, it is particularly flap and graft repairs that are most susceptible.

A 2004 survey of the American College of Mohs Micrographic Surgery and Cutaneous Oncology by Larson and Tayler found that between $7.5 \%$ and $17.8 \%$ of dermatologic surgeons used some form of intraoperative vital sign monitoring (29). They also conducted a prospective study to evaluate whether intraoperative vital sign monitoring could detect or prevent adverse events in cutaneous operations. In their patient population, blood pressure was always within $10 \%$ of baseline, and by the end of the cutaneous operation, it was less than $1.5 \%$ from baseline; the patients did not have adverse events. Larson and Taylor thus did not recommend routine intraoperative vital sign monitoring.

Alcalay and colleagues performed a similar study of blood pressure during MMS in 2005 (30). For 121 patients, they measured blood pressure at five time points throughout MMS. Findings included an actual drop in average blood pressure from $152 \pm 2 / 85 \pm 1 \mathrm{mmHg}$ at baseline to $139 \pm 2 / 79 \pm 1 \mathrm{mmHg}$ by the end of the procedure. Outcomes were the same regardless of hypertensive or normotensive initial blood pressure, prompting the authors to conclude that elevated blood pressure levels should not be the basis for deferring cutaneous surgery.

Dzubow (28), as well as Larson and Taylor (29), alleviated concerns over whether or not the use of lidocaine plus epinephrine for local anesthesia adversely affects blood pressure for typical dermatologic surgery. Dzubow studied 75 sequential Mohs patients and observed a statistically significant drop in the mean postanesthetic blood pressure $(115 \pm 12 \mathrm{mmHg})$ as compared with the mean preanesthetic blood pressure $(122 \pm 11 \mathrm{mmHg})$ in patients with hypertension. Although epinephrine predominantly produces beta $(\beta)$ and alpha $(\alpha)$ adrenergic effects, Dzubow notes that minute systemic levels of epinephrine are known to produce a $\beta$-vasodilatory effect with a resultant decrease in blood pressure. This is due to the greater sensitivity of the $\beta$-vasodilatory receptors to low blood concentrations of epinephrine than of the a-vasoconstrictor vascular receptors. Larson and Taylor studied the affect of local anesthetic (1\% lidocaine plus 1: 100,000 epinephrine) on vital signs and found the average deviations from baseline (positive or negative) for systolic pressure was $3.7 \%$ and for diastolic pressure 1.8\% (an insignificant relationship).

In a meta-analysis of 30 observational studies, Howell and colleagues calculate an odds ratio of 1.35 (1.17-1.56) for the association of hypertension with perioperative cardiac outcomes (e.g., perioperative ischemia, arrhythmia, cardiovascular lability, and cerebrovascular accident) in the setting of general anesthesia and invasive operation (31). They found little evidence for increased perioperative complications when admission blood pressures were less than $180 \mathrm{mmHg}$ systolic or $110 \mathrm{mmHg}$ diastolic. In this situation, they advocate continuation of the planned surgical procedure. Extrapolating to cutaneous surgery, it is a 
reasonable hypothesis that the cardiovascular risks associated with dermatologic surgery are much lower than those associated with general anesthesia and a more invasive operation. One dermatologic surgery group uses $200 \mathrm{mmHg}$ systolic and/or $100 \mathrm{mmHg}$ diastolic as their cutoff for starting serial 5-10 minute blood pressure monitoring; the procedure is deferred if the blood pressure does not decrease (32). Lastly, Shimizu and colleagues state in their study evaluating multiple antithrombotic agent use that no associations were observed between hemorrhagic complications and pre- or postoperative blood pressures (26). They cite blood pressure ranges preoperatively from 82 to $200 / 40$ to $110 \mathrm{mmHg}$ and postoperatively from 78 to $200 / 42$ to $110 \mathrm{mmHg}$ for the 756 patients with no bleeding complications. Although limited by not knowing the total number of patients that were hypertensive and to what degree, there clearly is a range of blood pressures within which the surgeons comfortably operated without hemorrhagic complications. Based on the above information, the following guidelines can be proposed:

1. For patients with a blood pressure $180 \mathrm{mmHg}$ systolic or $100 \mathrm{mmHg}$ diastolic or lower, and no other medical contraindications, cutaneous surgery may proceed. Literature noted above affirms that any potential perioperative cardiovascular complication risks are at a minimum, but there may be some unmeasured risk of postoperative wound bleeding or hematoma formation if the patient is hypertensive (140 $\mathrm{mmHg}$ systolic and $90 \mathrm{mmHg}$ diastolic).

2. For patients with blood pressure $181-200 \mathrm{mmHg}$ systolic or $101-110 \mathrm{mmHg}$ diastolic, cutaneous surgery may proceed depending on surgeon preference. If surgery does proceed, there may be a small risk of cardiovascular lability. One cautionary measure that can be taken by dermatologic surgeons performing cutaneous surgery is basic life support and/or advanced cardiac life support training. One survey found 75\% of Mohs surgeons maintained basic life support certification and 50\% maintained advanced cardiac life support certification (29).

3. For patients with blood pressure exceeding $200 \mathrm{mmHg}$ systolic or $110 \mathrm{mmHg}$ diastolic, it may be prudent to defer the surgical procedure until after the patient is properly evaluated by his or her primary medical physician, especially if the procedure is elective; however, there is no specific evidence quantifying the operative risk at these blood pressures.

Finally, it is important to remember that anxiety over the procedure and/or the "white-coat" phenomenon may also cause a patient's blood pressure to rise. Speaking in a calm, reassuring fashion during surgery ("talkesthesia"), playing relaxing music, and when necessary, prescribing mild anxiolytics, may help mitigate these additional dynamics that are at play during surgery.

\section{Medical comorbidities}

There are several medical conditions pertinent to intraoperative and postoperative hemorrhage risk in cutaneous surgery that must be considered. In essence, it is most important to discern whether a patient has an underlying bleeding tendency that can be congenital or acquired. Several lines of questioning can screen for such issues: Do you have any known bleeding problems? Do you have any known diseases that cause you to bleed 
easily? Have you ever experienced uncontrollable bleeding during or after a medical procedure? Does anyone in your family have problems with excessive bleeding? Do you have any liver disease or history of liver transplantation? Have you ever been diagnosed with a low platelet count (or thrombocytopenia)? How much alcohol do you drink? If such questioning or physical signs of easy bruising during the preoperative evaluation suggest a bleeding abnormality, one can consider ordering screening lab tests, such as complete blood count, prothrombin time, and partial thromboplastin time, to help identify an underlying disorder.

Peterson and Joseph describe their MMS encounter with a 78-year-old Caucasian man with previously known congenital factor IX deficiency and review inherited bleeding disorders in dermatologic surgery (33). They divide possible acquired or inherited defects in hemostasis into four categories of causes: (i) decreased vasoconstriction (e.g., older age, skin atrophy, chronic steroid use, Ehlers-Danlos syndrome and Osler-Weber-Rendu syndrome); (ii) decreased platelet adhesion and aggregation (e.g., vonWillebrand disease, osteogenesis imperfecta, Marfan syndrome); (iii) decreased coagulation (e.g., factor I-XIII deficiencies); and (iv) increased fibrinolysis (e.g., liver transplantation, disseminated intravascular coagulation, and inherited fibrinolytic abnormalities). Both chronic liver and kidney disease can also affect platelet function, and patients with such conditions may display an increased risk of bleeding or "oozing" during surgery.

The three most common congenital coagulation defects are von Willebrand disease (affects $1 \%$ of the population), factor VIII deficiency (hemophilia A), and factor IX deficiency (hemophilia B) (33). Based on experience with their patient having factor IX deficiency, Peterson and Joseph described five essential steps to successful cutaneous operation in patients with inherited bleeding disorders: (i) consult a hematologist to help manage the patient care, including provision of a nurse to perform factor replacement; (ii) consider appropriate purified and recombinant coagulation factor replacement; (iii) maintain meticulous operative technique; (iv) consider reconstructive options that minimize undermining and potential bleeding risks without compromising an aesthetic or functional repair; (v) have close postoperative follow-up with the patient.

The single largest social factor that may affect bleeding tendency is alcohol consumption. It is believed that the purported cardiovascular benefit of mild to moderate consumption of alcohol daily is directly due to alteration of hemostasis. In their 2005 review on the effect of alcohol on hemostasis, Salem and Laposata highlight four major mechanisms by which alcohol inhibits platelets, decreases coagulation, and increases fibrinolysis (34). First, alcohol inhibits the platelet aggregation response to collagen and adenosine diphosphate. Through increasing intracellular calcium, alcohol also partially activates platelets; however, in this partially activated state, the platelets function poorly. Second, alcohol impairs coagulation via decreased fibrinogen, von Willebrand factor, and factor VII. Third, moderate alcohol use activates tissue plasminogen activator, but not plasminogen activator inhibitor-1 (PAI-1), leading to increased fibrinolysis. Finally, alcohol is a potent vasodilator that can exacerbate these effects with regards to hemostasis. It is therefore imperative for dermatologic surgeons to address alcohol consumption with their patients before and after 
cutaneous surgery; ideally, patients should avoid alcohol consumption 48 hours before and after surgery.

\section{Anatomy}

The majority of cutaneous operations performed by Mohs surgeons will be on lesions located on the head or neck. In the prospective study by Cook and Perone, $86.3 \%$ (1172/1358) of cases were located on the head or neck (2). Similarly, 85.5\% (3368/3937) of cases evaluated by Kimyai-Asadi and colleagues were of lesions located on the head and neck (3). This data reinforces the importance of knowing and understanding the anatomy of the head and neck areas with high vascularity. Robinson cites three "danger zones" for arterial bleeding in procedural dermatology, all located on the head: (i) the frontal branch of the temporal artery located at the temple; (ii) the facial artery at its crossing over the mandibular rim; and (iii) the angular artery adjacent to the nose (35). Only by knowing this anatomy well can the surgeon make incisions of the proper depth and undermine in the correct planes so as to minimize bleeding complications.

\section{Intraoperative management of hemorrhage}

One of the first steps taken to help minimize intraoperative bleeding is the addition of epinephrine to the local anesthetic of choice. Most often, the amide anesthetic lidocaine $(0.5-2.0 \%)$ is used in cutaneous surgery. The concentration of epinephrine added to the local anesthetic is usually either 1: 100,000 or 1: 200,000, and provides three major benefits: prolongs activity of the anesthetic, decreases volume of anesthetic required, and via local vasoconstriction, it decreases risk of intraoperative bleeding (36). It is the latter benefit that prompts cutaneous surgeons to so frequently utilize epinephrine in local anesthesia. Without epinephrine, transected vessels vasodilate after the initial spasm/vasoconstriction period, thereby allowing for intraoperative hemorrhage (37). Contraindications to epinephrine use are uncontrolled hyperthyroidism and pheochromocytoma (36). Ultimately, when using epinephrine, it is sensible to wait 15-20 minutes after anesthetic injection before initiating surgery to allow for the maximum vasoconstrictive benefits.

Even before a procedure is started, a few minor but critical steps can be taken to assure safe and efficient surgery: proper positioning of the patient at a slight incline such that the surgical area is both above the heart (if possible) and easily accessible for the surgeon (while remaining ergonomically comfortable); using good lighting to allow for maximum visibility. There are three basic intraoperative steps in cutaneous surgery that potentially generate hemorrhagic complications - initial incision, extirpation of the skin lesion, and undermining of tissue adjacent to the wound. As alluded to above, understanding cutaneous anatomy allows for correct incision depths so as to avoid major arteries (e.g., temporal, facial, and angular arteries) or veins. Once an incision (usually an ellipse for basic excision or circle or oval for MMS) has been made completely surrounding a skin lesion to the level of the subcutaneous fat or fascia, the lesion of concern can be removed. It is important to perform even and uniform extirpation so as to avoid unnecessary severing of deeper vessels (or nerves) and to facilitate wound closure. Undermining of the tissue adjacent to the surgical defect is necessary to generate both vertical and horizontal movement of the wound edges and involves stretching and/or breaking the interlobular collagenous septae that connect the 
deep fascia with the reticular dermis (38). Boyer et al. describe several benefits to undermining, including decreased wound closing tension, reduction of tissue redundancies, and wider distribution of wound contraction/scar forces. All of these benefits ultimately aid wound healing and final cosmetic outcome. The major risk with undermining, however, is the severing of vessels and the risk of subsequent hematoma formation.

Goldman divides intraoperative hemorrhage into two major categories: "oozing" and frank "bleeding." (39) He further subdivides "oozing" into superficial/dermal oozing and deep oozing, the latter more commonly seen due to muscular injury. Frank bleeding can be arterial/venous or arteriolar/venular. It is up to the surgeon to evaluate the surgical site and decide the quality of the hemorrhage and likely etiology. There are several different options of management depending on the quality and etiology of the hemorrhage. For superficial/ dermal oozing, manual pressure, electrosurgery (electrodessication or electrocoagulation), heat cautery (electrocautery), or topical styptics are all methods that can stop the oozing (39). Electrosurgery and heat cautery work via protein denaturation and do not initially depend upon the clotting cascade. The most common styptics (also known as topical caustic hemostatics) used are aluminum chloride (favored since it is least likely to leave pigment), $20 \%$ ferric sulfate (Monsel's solution), and 10-50\% silver nitrate (37). If the oozing is deeper and more muscular, the same techniques of pressure and/or electrosurgery may be used to control the hemorrhage.

One additional option available to help control deeper oozing is the use of non-caustic topical hemostatic agents. Achneck and colleagues recently published a comprehensive review of noncaustic topical hemostatic agents in the general surgery setting (40). They divide the agents into five major product types: physical agents (e.g., Ostene ${ }^{\circledR}$, watersoluble alkylene oxide copolymers, Ceremed, Los Angeles, CA, USA), absorbable agents (e.g., Surgicel®, oxidized regenerated cellulose, Ethicon, Inc., Somerville, NJ, USA), biologic agents (e.g., Tisseel®, fibrin sealant, Baxter, Deerfield, IL), synthetic agents (e.g., Dermabond ${ }^{\circledR}$, Ethicon, Inc.), and hemostatic dressings (e.g., HemCon ${ }^{\circledR}$ Bandages, chitosan, HemCon Medical Technologies Inc., Portland, OR, USA). Many of these topical hemostatic agents are applicable in dermatologic surgery - those that are most practical and costeffective on a routine basis are gelatin foams (e.g., Gelfoam ${ }^{\circledR}$ (absorbable gelatin sponge) and Gelfilm ${ }^{\circledR}$ (absorbable gelatin film), Pfizer), oxidized cellulose (e.g., Surgicel), and microfibrillar collagen (e.g., Instat ${ }^{\circledR}$, Ethicon, Inc.) (37). These agents provide a scaffold on which coagulation and platelet activation can occur. These hemostatic agents are used in dermatologic surgery in situations to prevent and/or stop bleeding from primary defects left to heal by granulation/secondary intention, to place on defects being bandaged for delayed flap or graft closure, or around the stalks of pedicled flaps to minimize postoperative bleeding.

Smaller arteriolar/venular hemorrhage is best managed using electrosurgery (electrodessication and electrocoagulation) or heat cautery. Regardless, any electrosurgery should be precise to minimize excessive and unnecessary charring of surrounding tissue. Larger arterial or venous bleeding requires ligation of the vessels using absorbable sutures. In order to perform either electrosurgery or suture ligation, the surgeon first must be able to visualize and isolate the source of the bleeding. Seeing a pool of blood in the operative field 
can generate anxiety; however, deep breaths and maintaining a sense of calm will help the surgeon systematically assess the best way to manage the bleeding. It will also reassure the patient who in most dermatologic procedures is awake and alert! Methods to improve visibility of a bloody surgical field include: the use of skin hooks to raise skin edges nontraumatically and check for bleeding distant to the incision or under a flap; gauze and/or cotton-tip applicators (41); manual pressure outside the wound but proximal to the bleeding vessel; additional undermining to increase access to a culprit vessel; and if a rapid bleed, a suction device may be needed. One or more of these methods may be required for a single patient. After ensuring visibility of and access to the bleeding vessel, one of several suturing techniques may be used if ligation of the vessel is required. The simplest and most commonly used is the figure-of-eight stitch. It is important to locate and suture both ends of a transected blood vessel. There are also a variety of suturing techniques, include the horizontal mattress suture, which a surgeon may find useful for particular wounds $(37,42)$. Finally it is critical to suture and close any dead space to minimize the risk of hematoma formation. For the types of hemorrhage encountered during cutaneous operation, numerous options to halt the bleeding exist as described above. Just because a patient experiences heightened intraoperative bleeding, it does not mean they are on blood thinners; West and colleagues via their study discount the notion that cutaneous surgeons can predict blood thinner use by intraoperative bleeding (43). Occasionally, a surgeon will encounter a patient whose surgical wound oozes or continues to bleed despite multiple attempts at hemostasis. In such a case, it may be more practical to leave the wound partially open or consider placing a Penrose drain to allow for drainage rather than have blood accumulate. If a drain is used in the wound, it is important to remove it in a timely fashion (usually after 24 hours) to prevent an infection.

\section{Post-operative patient management}

Once the cutaneous operation is complete, whether simple elliptical excision or MMS, the final phase (i.e., the post-operative phase) of patient care and instructions begins. There are three key components to the post-operative period - bandaging the wound/surgical repair site, educating the patient and any family about proper care of the site while at home, and lastly, the part all cutaneous surgeons hope to avoid, rectifying any postoperative complications or patient concerns.

\section{Bandaging the surgical site}

Almost all surgical procedures end with the placement of a pressure dressing, with the goals that enough pressure is generated to provide additional hemostasis as the patient heads home, as well as protect the vulnerable new wound. A typical pressure dressing involves the placement of a non-adherent film (e.g., Telfa ${ }^{\mathrm{TM}}$, Kendall Brands, Covidien, Mansfield, MA, USA) directly on the operative site, followed by one or more layers of cotton gauze, and finally, securing the dressing with adhesive tape (2). There are numerous materials and methods for applying bandages; however, any single pressure dressing is probably not effective beyond 24 hours. In a study of elastic bandages applied to burn wounds, Sawada found that the pressure under the bandages decreases throughout a single day (44). Thus, it 
is generally appropriate to instruct patients to remove the pressure dressing the following morning or day.

\section{Patient instructions}

After the operation, every patient should be provided with verbal and written instructions on wound care, what to do if there is bleeding (either oozing or frank bleeding), activity levels, alcohol use, signs and symptoms of infection or wound dehiscence or necrosis, and ultimately, when to call the surgeon. One of the hardest instructions for a patient (particularly the younger and professionally active patients) to follow is to rest in the postoperative period. It is important for patients to minimize physical activity in order to avoid placing excessive wound tension on the surgical site. Avoiding any strenuous exercise, bending, lifting heavy objects, or physical activity that could potentially lead to direct trauma to the surgical site is critical to proper wound healing and prevention of hemorrhagic complications. In addition, sites which are in constant motion due to daily activities such as lips or the perioral area require specific instructions such as minimizing talking/laughing, eating soft or well-cooked foods, avoiding sucking action via a straw, and taking care when inserting or removing dentures.

\section{Managing hematoma and hemorrhage}

One of the most challenging aspects of outpatient surgery is assessing the status of wounds or worri-some complications with patients over the telephone. Although the age of digital cameras and email has made this easier, anticipating and communicating the expected initial postoperative appearance of wounds to the patient may help minimize unnecessary or alarming phone calls. Many surgical procedures on the face can lead to significant swelling and bruising (or ecchymosis), particularly around the eyes and lips. Due to gravity, the ecchymosis can often extend far beyond the incision site (see FIGS 1 and 2). It is essential to prepare the patient for this normal development and reassure them that the ecchymosis will resolve spontaneously and have no long-term consequence.

The risk for hemorrhagic complications is greatest in the first 48 hours after surgery. When a patient contacts a physician with concern for bleeding, the first step is to determine whether the bleeding represents mild postoperative oozing (due possibly to the waning of epinephrine's vasoconstrictive effects) or frank, persistent bleeding or the development of a hematoma. Having the patient remove the dressing to visualize the wound and asking a few simple questions can help elicit the problem: Is there continuous bleeding from underneath the pressure bandage? Is the pressure bandage soaked or saturated? Is there a painful swelling or expanding mass or firmness? If a hematoma has not already formed, the first step performed is by the patient at home - he or she or a family member applies direct, firm, pinpoint pressure over the surgical wound for 15-20 consecutive minutes while watching a clock. The details of this directive are key; oftentimes patients understandably get frightened or overwhelmed when they see bleeding from a surgical site and grab a large towel to press over the general area. The surgeon must calm the patient, have them try to identify the specific area from which there is bleeding and apply direct, steady pressure. If not actually watching a clock, the patient tends to overestimate the amount of time he or she actually holds pressure due to anxiety and fearful curiosity. The majority of minor oozing and 
bleeding from cutaneous surgery wounds will stop with such direct firm pressure. If bleeding continues despite this intervention (or, in our practice, two cycles of such an intervention), then patients are instructed to have the wound evaluated by the surgeon.

In Dorland's Medical Dictionary, a hematoma is defined as "a localized collection of blood, usually clotted, in an organ, space, or tissue, due to a break in the wall of a blood vessel" (45). A hematoma can be divided into four stages of evolution: early formation (stage I), gelatinous (stage II), organized (stage III), and liquefaction (stage IV) (37). Stage I hematomas are being formed by active bleeding into the wound and/or adjacent tissues; they often fill dead spaces formed from inadequate layered closures. Ideally, dermatologic surgeons likely will encounter hematomas in this early formation stage, since most patients (following the provided appropriate patient care instructions) will call with concerns over an expanding (potentially rapid), warm, and possibly painful mass. An expanding hematoma requires the patient to return to the surgical suite for evacuation and definitive hemostasis. Hematomas in the periorbital and cervical locations are considered dermatologic and medical emergencies because of the possible mass effect on underlying vital structures. The same mass effect can compromise blood supply to flaps and grafts and lead to dehiscence of wound edges and tissue necrosis (46). In addition, if not treated, a hematoma provides a happy medium for bacterial growth and increases infection risk.

The primary method to treat an expanding hematoma is: (i) partial or complete opening of the surgical wound, (ii) appropriate suture ligation or electrosurgery of the culprit vessel(s), and (iii) either full-layer reclosure of the wound or healing by secondary intention (if there is a high risk of more bleeding or the wound is contaminated) (See FIGS 3-8). One of the debates for dermatologic surgeons is whether to use plain lidocaine or lidocaine with epinephrine when reanesthetizing a bleeding surgical wound. The concern for some is that the addition of epinephrine will cause blood vessels to constrict and make it harder to pinpoint the bleeding culprits. However, in our opinion, it is usually helpful to have epinephrine on board for longer-lasting anesthesia for the patient. The surgeon should then take thorough measures to look carefully at all edges of the wound and irrigate it with saline to identify any problematic areas when the wound has been reopened. Finally, it is the general practice of most surgeons to place the patients on antibiotics after such an event to prevent a secondary wound infection.

During the gelatinous and organized stages, blood clots within hematomas and progresses to a firm and rubbery consistency; however, after 7-10 days, fibrinolysis leads to hematoma liquefaction. It is during this liquefactive stage that simple needle aspiration through the suture line of the hematoma may be considered. Singer and Dagum have reviewed management of subungual hematomas and advocate simple nail trephination with the use of portable cautery for most cases; nail removal or surgical repair is only performed when there is clear damage to the nail or nail folds (47).

\section{Conclusions}

This article discusses preoperative, intraoperative, and postoperative issues relating to hemorrhagic complications in cutaneous surgery (Refer to FIG. 9). While the overall 
complication risk is fortunately low with dermatologic surgery, it is important nonetheless to understand the factors that affect hemostasis. Complications and how we contend with them are the inevitable thorns of surgical procedures - they remind us of our humanity, and, ultimately, our resilience. To help minimize these unwanted events, the surgeon must be meticulous in every sense of the word and in every phase of the procedure.

\section{References}

1. Coldiron BM, Healy C, Bene NI. Office surgery incidents: what seven years of Florida data show us. Dermatol Surg. 2008; 34(3):285-291. discussion 91-2. [PubMed: 18177404]

2. Cook JL, Perone JB. A prospective evaluation of the incidence of complications associated with Mohs micrographic surgery. Arch Dermatol. 2003; 139(3):143-152. [PubMed: 12588220]

3. Kimyai-Asadi A, Goldberg LH, Peterson SR, Silapint S, Jih MH. The incidence of major complications from Mohs micrographic surgery performed in office-based and hospital-based settings. J Am Acad Dermatol. 2005; 53(3):628-634. [PubMed: 16198783]

4. Otley CC, Fewkes JL, Frank W, Olbricht SM. Complications of cutaneous surgery in patients who are taking warfarin, aspirin, or nonsteroidal anti-inflammatory drugs. Arch Dermatol. 1996; 132(3): 161-166. [PubMed: 8629823]

5. Alcalay J. Cutaneous surgery in patients receiving warfarin therapy. Dermatol Surg. 2001; 27(3): 756-758. [PubMed: 11493301]

6. Bartlett GR. Does aspirin affect the outcome of minor cutaneous surgery? Br J Plast Surg. 1999; 52(3):214-216. [PubMed: 10474474]

7. Billingsley EM, Maloney ME. Intraoperative and postoperative bleeding problems in patients taking warfarin, aspirin, and nonsteroidal antiinflammatory agents. A prospective study. Dermatol Surg. 1997; 23(5):381-383. discussion 4-5.

8. Kargi E, Babuccu O, Hosnuter M, Babuccu B, Altinyazar C. Complications of minor cutaneous surgery in patients under anticoagulant treatment. Aesthetic Plast Surg. 2002; 26(3):483-485. [PubMed: 12621574]

9. Syed S, Adams BB, Liao W, Pipitone M, Gloster H. A prospective assessment of bleeding and international normalized ratio in warfarin-anticoagulated patients having cutaneous surgery. $\mathrm{J}$ Am Acad Dermatol. 2004; 51(3):955-957. [PubMed: 15583588]

10. Lewis KG, Dufresne RG Jr. A meta-analysis of complications attributed to anticoagulation among patients following cutaneous surgery. Dermatol Surg. 2008; 34(2):160-164. discussion 4-5. [PubMed: 18093204]

11. Holmes DR Jr, Kereiakes DJ, Kleiman NS, Moliterno DJ, Patti G, Grines CL. Combining antiplatelet and anticoagulant therapies. J Am Coll Cardiol. 2009; 54(3):95-109. [PubMed: 19573725]

12. Grip L, Blomback M, Schulman S. Hypercoagulable state and thromboembolism following warfarin withdrawal in post-myocardial-infarction patients. Eur Heart J. 1991; 12(3):1225-1233. [PubMed: 1782954]

13. Palareti G, Legnani C. Warfarin withdrawal. Pharmacokinetic-pharmacodynamic considerations. Clin Pharmacokinet. 1996; 30(3):300-313. [PubMed: 8983860]

14. Schanbacher CF, Bennett RG. Postoperative stroke after stopping warfarin for cutaneous surgery. Dermatol Surg. 2000; 26(3):785-789. [PubMed: 10940066]

15. Alam M, Goldberg LH. Serious adverse vascular events associated with perioperative interruption of antiplatelet and anticoagulant therapy. Dermatol Surg. 2002; 28(11):992-998. discussion 8. [PubMed: 12460291]

16. Kovich O, Otley CC. Thrombotic complications related to discontinuation of warfarin and aspirin therapy perioperatively for cutaneous operation. J Am Acad Dermatol. 2003; 48(3):233-237. [PubMed: 12582394]

17. Kirkorian AY, Moore BL, Siskind J, Marmur ES. Perioperative management of anticoagulant therapy during cutaneous surgery: 2005 survey of Mohs surgeons. Dermatol Surg. 2007; 33(3): 1189-1197. [PubMed: 17903151] 
18. Kovich O, Otley CC. Perioperative management of anticoagulants and platelet inhibitors for cutaneous surgery: a survey of current practice. Dermatol Surg. 2002; 28(3):513-517. [PubMed: 12081682]

19. Wahl MJ. Dental surgery in anticoagulated patients. Arch Intern Med. 1998; 158(3):1610-1616. [PubMed: 9701094]

20. Fleisher LA, Beckman JA, Brown KA, et al. ACC/AHA 2007 guidelines on perioperative cardiovascular evaluation and care for noncardiac surgery: executive summary: a report of the American College of Cardiology/American Heart Association Task Force on Practice Guidelines (Writing Committee to Revise the 2002 Guidelines on Perioperative Cardiovascular Evaluation for Noncardiac Surgery): developed in collaboration with the American Society of Echocardiography, American Society of Nuclear Cardiology, Heart Rhythm Society, Society of Cardiovascular Anesthesiologists, Society for Cardiovascular Angiography and Interventions, Society for Vascular Medicine and Biology, and Society for Vascular Surgery. Circulation. 2007; 116(3): 1971-1996. [PubMed: 17901356]

21. Kramer E, Hadad E, Westreich M, Shalom A. Lack of complications in skin surgery of patients receiving clopidogrel as compared with patients taking aspirin, warfarin, and controls. Am Surg. 2010; 76(3):11-14. [PubMed: 20135932]

22. Fessenden JM, Wittenborn W, Clarke L. Gingko biloba: a case report of herbal medicine and bleeding postoperatively from a laparoscopic cholecystectomy. Am Surg. 2001; 67(3):33-35. [PubMed: 11206893]

23. Beckert BW, Concannon MJ, Henry SL, Smith DS, Puckett CL. The effect of herbal medicines on platelet function: an in vivo experiment and review of the literature. Plast Reconstr Surg. 2007; 120(3):2044-2050. [PubMed: 18090773]

24. Chang LK, Whitaker DC. The impact of herbal medicines on dermatologic surgery. Dermatol Surg. 2001; 27(3):759-763. [PubMed: 11493302]

25. Collins SC, Dufresne RG Jr. Dietary supplements in the setting of mohs surgery. Dermatol Surg. 2002; 28(3):447-452. [PubMed: 12081669]

26. Shimizu I, Jellinek NJ, Dufresne RG, Li T, Devarajan K, Perlis C. Multiple antithrombotic agents increase the risk of postoperative hemorrhage in dermatologic surgery. J Am Acad Dermatol. 2008; 58(3):810-816. [PubMed: 18423258]

27. Straith RE, Raju DR, Hipps CJ. The study of hematomas in 500 consecutive face lifts. Plast Reconstr Surg. 1977; 59(3):694-698. [PubMed: 850705]

28. Dzubow LM. Blood pressure as a parameter in dermatologic surgery. Arch Dermatol. 1986; 122(3):1406-1407. [PubMed: 3789773]

29. Larson MJ, Taylor RS. Monitoring vital signs during outpatient Mohs and post-Mohs reconstructive surgery performed under local anesthesia. Dermatol Surg. 2004; 30(3):777-783. [PubMed: 15099324]

30. Alcalay J, Alkalay R, Grossman E. Blood pressure levels decrease during Mohs micrographic surgery. J Drugs Dermatol. 2005; 4(3):469-470. [PubMed: 16004020]

31. Howell SJ, Sear JW, Foex P. Hypertension, hypertensive heart disease and perioperative cardiac risk. Br J Anaesth. 2004; 92(3):570-583. [PubMed: 15013960]

32. Wilde J, Hauser D, Leshin B. Perioperative care of the surgical patient. Semin Cutan Med Surg. 2004; 23(3):203-206. [PubMed: 15584686]

33. Peterson SR, Joseph AK. Inherited bleeding disorders in dermatologic surgery. Dermatol Surg. 2001; 27(3):885-889. [PubMed: 11722527]

34. Salem RO, Laposata M. Effects of alcohol on hemostasis. Am J Clin Pathol. 2005; 123(Suppl.):S96-S105. [PubMed: 16100871]

35. Robinson, JK. Anatomy for procedural dermatology. In: Robinson, JK.; Hanke, CW.; Siegel, DM.; Fratila, A., editors. Surgery of the skin. Moby Elsevier; New York, NY: 2010. p. 3-27.

36. Hruza, GJ. Anesthesia. Dermatology. 2nd ed.. Bolognia, JL.; Jorrizo, JL.; Rapini, RP., editors. Mosby Elsevier; Span: 2008. p. 2173-2181.

37. Nguyen, TH.; Erickson, QL. Hemostasis. Surgery of the skin. 2nd ed.. Robinson, JK.; Hanke, CW.; Siegel, DM.; Fratila, A., editors. Mosby Elsevier; New York, NY: 2010. p. 225-238. 
38. Boyer JD, Zitelli JA, Brodland DG. Undermining in cutaneous surgery. Dermatol Surg. 2001; 27(3):75-78. [PubMed: 11231251]

39. Goldman, G. Wound closure materials and instruments. In: Bolognia, JL.; Jorizzo, JL.; Rapini, RP., editors. Dermatology. 2nd ed.. Mosby Elsevier; Spain: 2008. p. 2183-2193.

40. Achneck HE, Sileshi B, Jamiolkowski RM, Albala DM, Shapiro ML, Lawson JH. A comprehensive review of topical hemostatic agents: efficacy and recommendations for use. Ann Surg. 2010; 251(3):217-228. [PubMed: 20010084]

41. Orengo I, Salasche SJ. Surgical pearl: the cotton-tipped applicator-the ever-ready, multipurpose superstar. J Am Acad Dermatol. 1994; 31(3):658-660. [PubMed: 8089294]

42. Harahap M, Siregar AS. Method of minimizing hemorrhage in excising hemangioma. J Dermatol Surg Oncol. 1990; 16(3):442-444. [PubMed: 2187915]

43. West SW, Otley CC, Nguyen TH, et al. Cutaneous surgeons cannot predict blood-thinner status by intraoperative visual inspection. Plast Reconstr Surg. 2002; 110(3):98-103. [PubMed: 12087237]

44. Sawada Y. Alterations in pressure under elastic bandages: experimental and clinical evaluation. J Dermatol. 1993; 20(3):767-772. [PubMed: 8120239]

45. Anderson, DM.; Keith, J.; Novak, PD.; Elliot, MA. Dorland's illustrated medical dictionary. W.B. Saunders Company; Philadelphia, PA: 1994.

46. Talboy, GE., Jr; Gordon, I. Wounds and wound healing. Essentials of general surgery. In: Lawrence, PF., editor. 4th ed.. Lippincott Williams \& Wilkins; New York, NY: 2006. p. 147-161.

47. Singer AJ, Dagum AB. Current management of acute cutaneous wounds. N Engl J Med. 2008; 359(3):1037-1046. [PubMed: 18768947] 


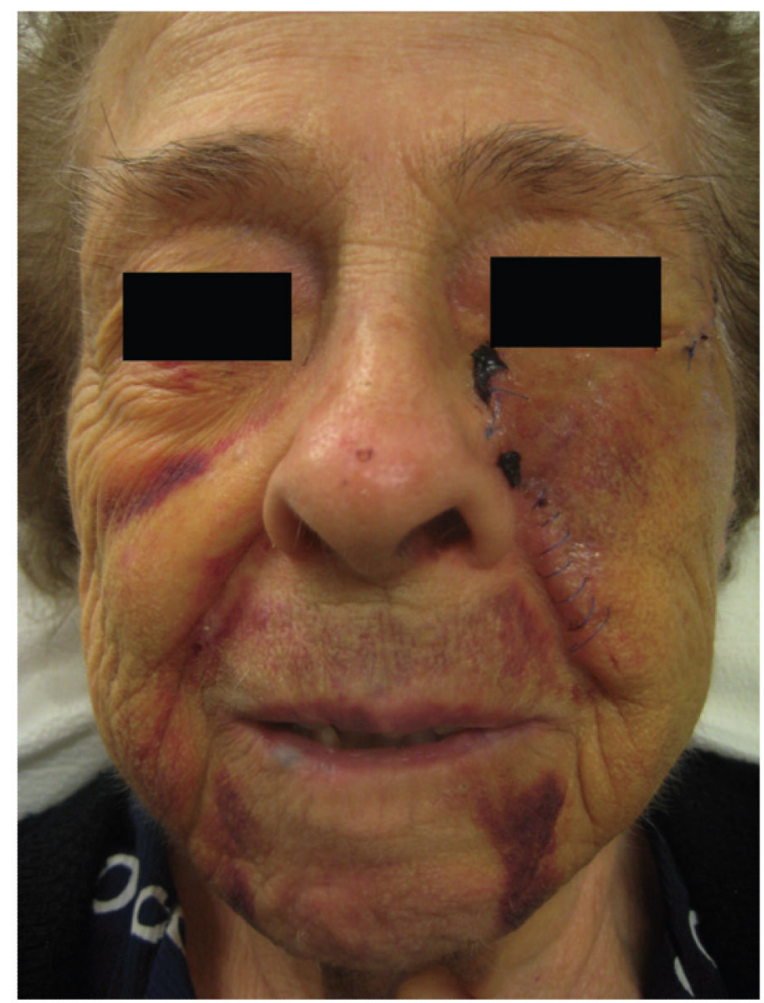

FIG. 1. This patient had a cheek advancement flap performed on the left side of her face. However, she developed bruising bilaterally, demonstrating that ecchymosis from such procedures can be quite extensive 


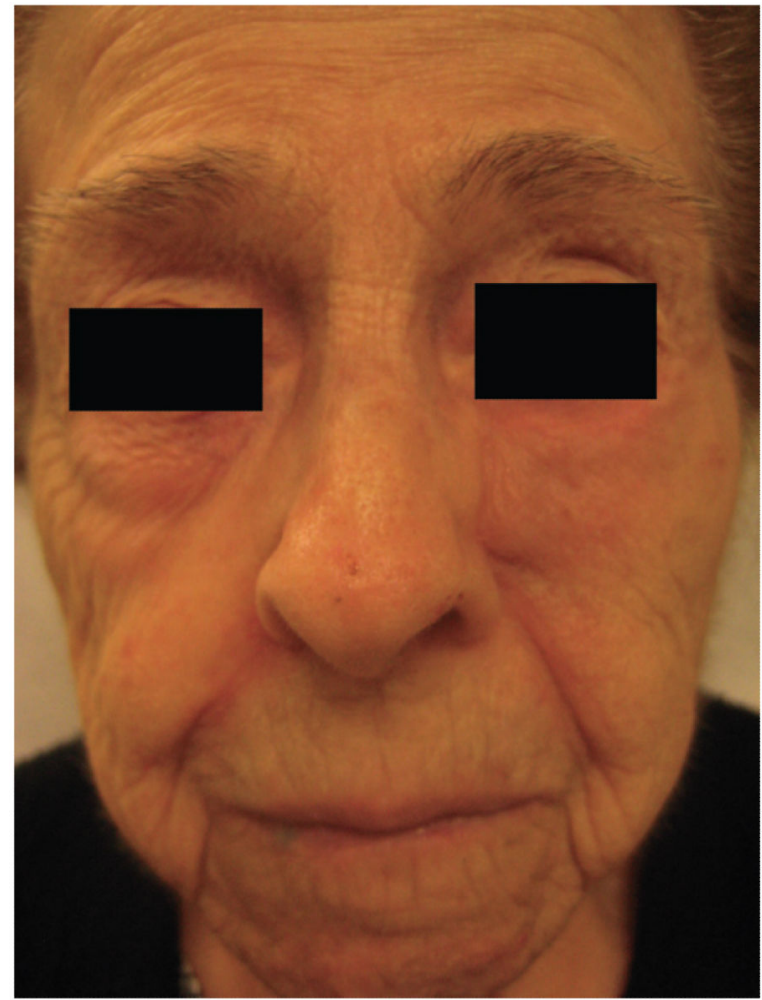

FIG. 2. After a few weeks, the ecchymosis has resolved without any adverse consequences 


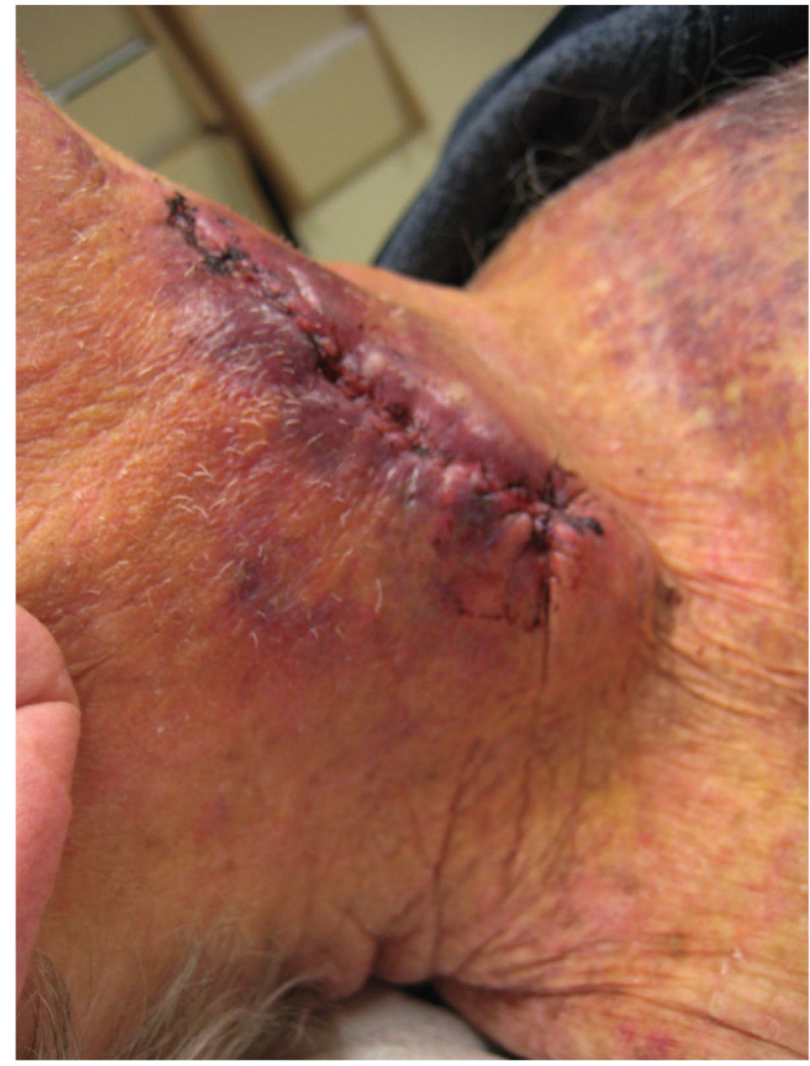

FIG. 3. A hematoma has developed under this linear repair on the neck 


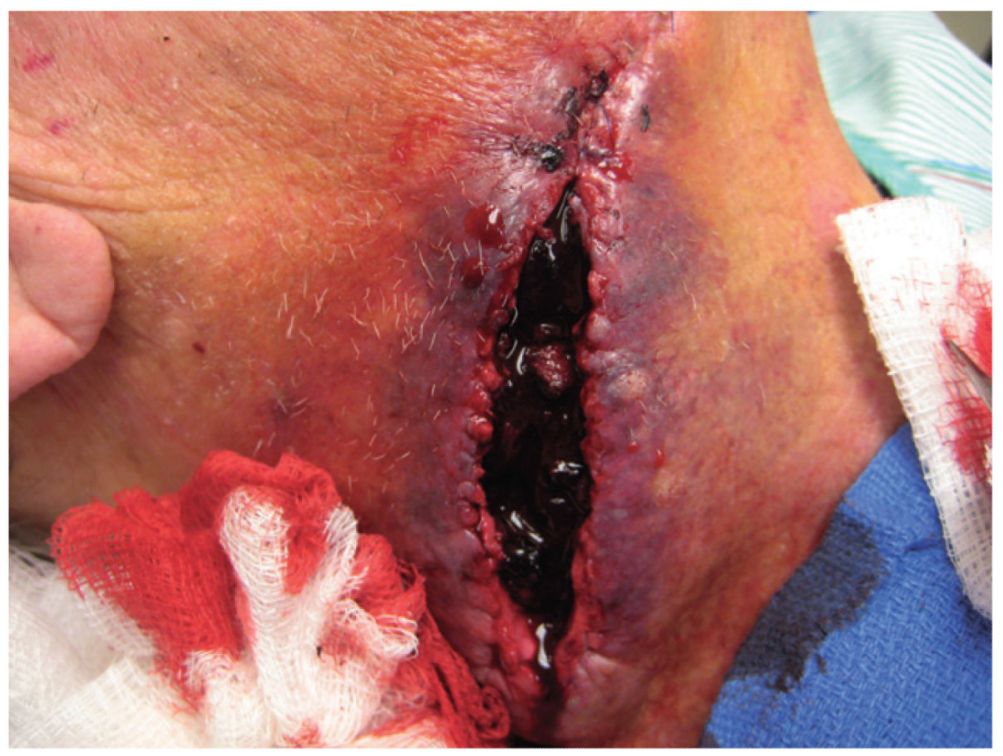

FIG. 4. Due to its expansive nature and critical location, the neck wound is opened, revealing large recently formed clots 


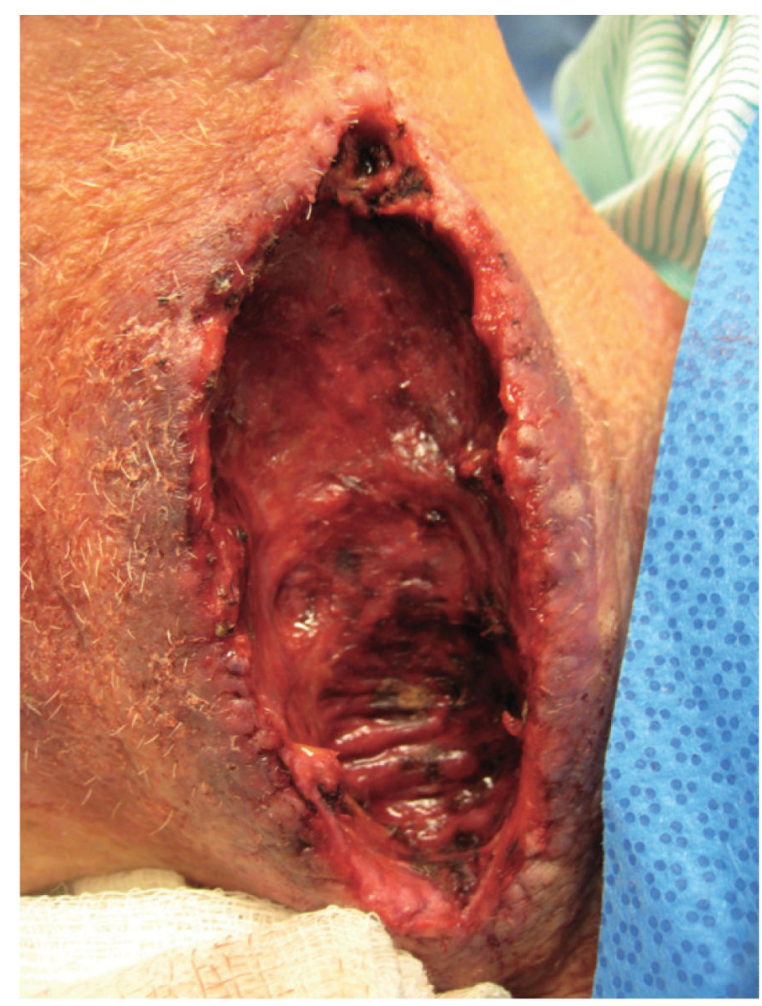

FIG. 5. The hematoma is evacuated, hemostasis is achieved, and the wound is then thoroughly irrigated with sterile saline to expose any inconspicuous areas of bleeding and confirm that hemostasis has been thoroughly achieved 


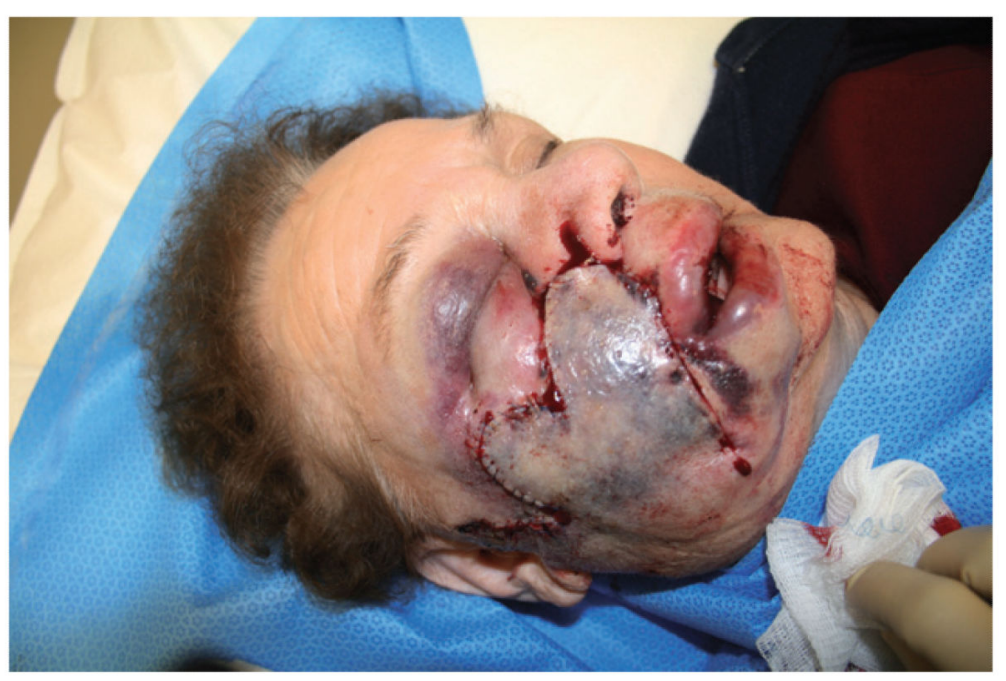

FIG. 6. A hematoma developed postoperatively under this large flap when the patient opened her mouth widely to remove her dentures. Note the tension as well as the bluish hue that the hematoma is creating under the flap. The wound was opened and hemostasis was reattempted 


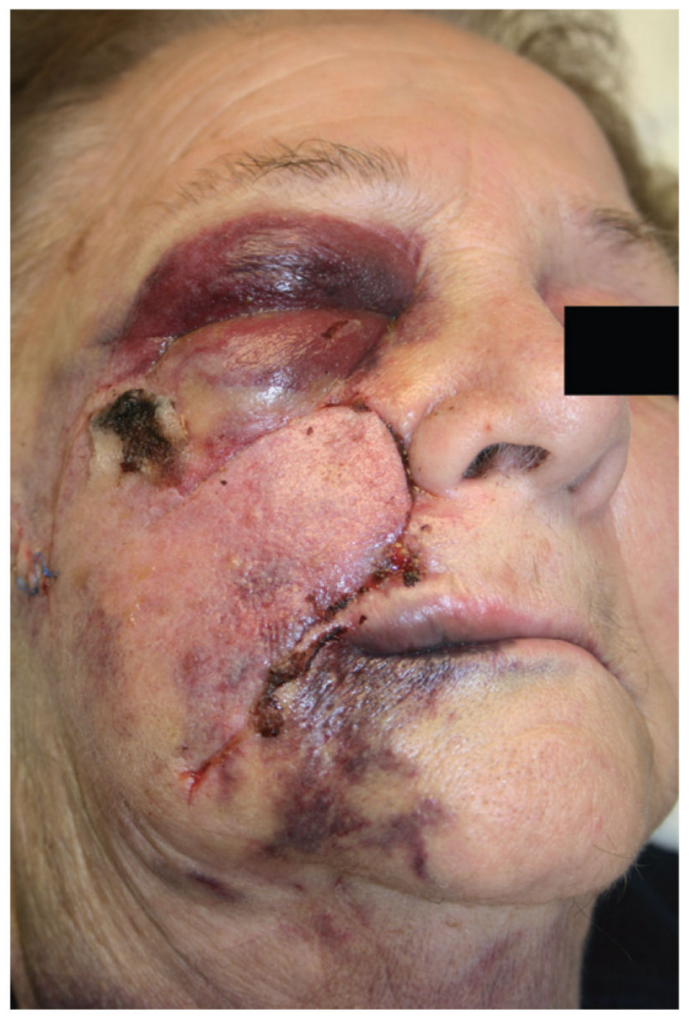

FIG. 7. The patient was reevaluated in 24 hours. This follow-up is critical when managing large hematomas. Notice that the wound has been resutured. Surgicel (a noncaustic topical hemostatic agent) is visible at the superior portion of the flap 


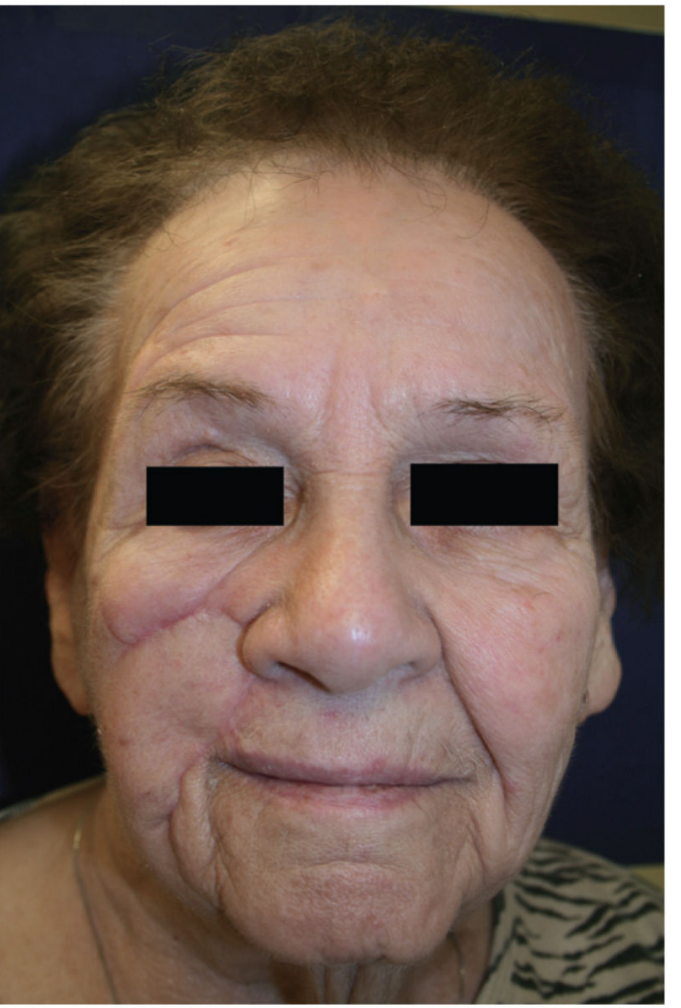

FIG. 8. The patient at several weeks later is continuing to heal well without any long-term adverse consequences 


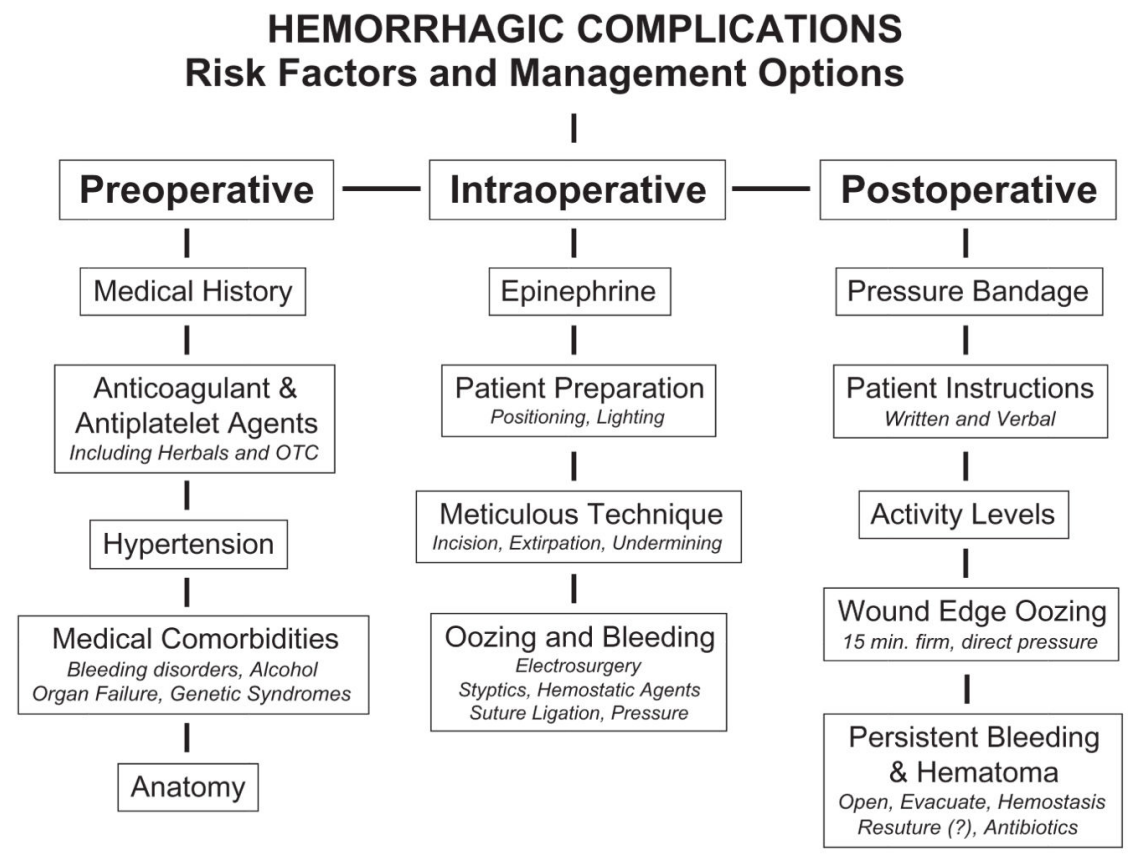

FIG. 9. Schematic of the different preoperative, intraoperative, and postoperative factors that affect the risk of hemorrhagic complications and management options 